\section{In response (2)}

We thank Wallis and Guptha for sharing their audit results. Their results show that the majority of their patients were admitted with an infection, which was also stated as the cause of death. Our results revealed that infection was the primary reason for admission in $32 \%$ of the cohort. ${ }^{1}$ Symptoms arising as a result of infection can be similar to those of someone who is dying, and as healthcare professionals working in an environment which focuses on cure, we sometimes treat infections without recognising that the patient is actually dying from their underlying illness. ${ }^{2-4}$

However, we fully agree that the treatment of an infection can be entirely appropriate for patients who may be entering their last days of life - either for symptom control or because the prognosis is uncertain (especially in those with non-malignant disease who have unpredictable disease trajectories). The current version of the Liverpool Care Pathway (LCP) for the dying does not stipulate that all antibiotics should be stopped, but that inappropriate antibiotics should be discontinued. ${ }^{5}$ Thus in those patients where appropriate, it is possible for the patient to be on the LCP while receiving antibiotics. However, as suggested by our audit and the work of others, the 'diagnosis of dying' can be difficult to make and thus the 'right time' to place a patient onto the LCP or other end-of-life care pathway can be challenging. We therefore advocate that until sensitive and specific prognostication tools are available, as stated above, we should 'assist clinicians in identifying those patients who might die during their current hospital admission thereby enabling active treatment where appropriate alongside symptom relief'. ${ }^{1}$ This approach would enable those patients who require appropriate antibiotics to receive them, while allowing 'healthcare professionals to allay their own anxieties in instances of clinical uncertainty?

JANE GIBBINS

Specialist Registrar in Palliative Medicine

RACHEL MCCOUBRIE

Consultant in Palliative Medicine

KAREN FORBES

Macmillan Professorial Teaching Fellow and Consultant in Palliative Medicine

University Hospitals of Bristol NHS Foundation Trust

\section{References}

1 Gibbins J, McCoubrie R, Alexander N, Kinzel C, Forbes K. Diagnosing dying in the acute hospital setting; are we too late? Clin Med 2009;4:116-9.

2 SUPPORT Principle Investigators. A controlled trial to improve care for seriously ill hospitalised patients: the study to understand prognoses and preferences for outcomes and risks of treatment. JAMA 1995;274:1591-8.

3 Ellershaw J, Ward C. Care of the dying patient: the last hours or days of life. BMJ 2003;326:30-4.

4 Miller F, Fins J. A proposal to restructure hospital care for dying patients. $\mathrm{N} \mathrm{Engl}$ Med 1996;334:1740-2.

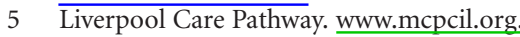
uk/liverpool_care_pathway

\section{Investigation of the patient with pleural effusion (1)}

Editor - Rahman and Munavvar's paper on investigating the patient with pleural effusion (Clin Med April 2009 pp 174-8) made no mention of the utility of quantifying pleural fluid adenosine deaminase (ADA) as a diagnostic parameter. Assay of ADA is simple and inexpensive, coupled with a relatively high sensitivity and specificity in identifying tuberculous pleuritis, enhanced further when the effusion in question is richly lymphocytic. ${ }^{1,2}$ While the positive predictive value of ADA may be lowered by its use in areas of low tuberculosis (TB) prevalence, its negative predictive value should remain unaffected, retaining its overall value as a subsidiary 'rule out' test for suspected TB pleural disease. ${ }^{3}$ Furthermore, ADA levels falling below the diagnostic cut-off threshold (around $40 \mathrm{U} / \mathrm{l}$ ) have been shown to virtually exclude TB as a cause of pleural effusion even when the collection is lymphocytic. ${ }^{3,4}$ We suspect that many physicians continue to find the analysis of ADA useful in the setting of suspected TB pleural sepsis while eagerly awaiting the emergence of newer immune-based tests of pleural fluid.

FELIX CHUA

Consultant Thoracic Physician

JANICE GROTH

Senior TB Specialist Nurse,

St George's Healthcare NHS Trust, London

\section{References}

1 Valdes L, Alvarez D, San Jose E et al. Value of adenosine deaminase in the diagnosis of tuberculous pleural effusion in young patients in a region of high prevalence of tuberculosis. Thorax 1995;50:600-3.

2 Burgess LJ, Maritz FJ, Le Roux I et al. Combined use of adenosine deaminase with lymphocyte/neutrophil ratio. Chest 1996;109:414-9.

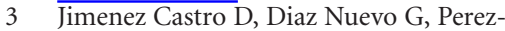
Rodriguez E, Light RW. Diagnostic of adenosine deaminase in nontuberculous lymphocytic pleural effusions. Eur Respir J 2003;21:220-4.

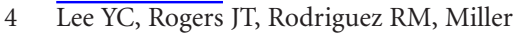
KD, Light RW. Adenosine deaminase levels in nontuberculous lymphocytic pleural effusions. Chest 2001;120:356-61.

\section{Investigation of the patient with pleural effusion (2)}

Given the fact that culture of pleural fluid (using Lowenstein medium) has only 36.6\% sensitivity for diagnosis of tuberculous pleural effusion versus $79.8 \%$ sensitivity obtained with the more invasive modality of histological identification of caseating granuloma, ${ }^{1}$ among the non-invasive 'special tests' for evaluation of pleural effusion, ${ }^{2}$ mention should also have been made of adenosine deaminase (ADA) ${ }^{3}$ and interferon-gamma (IFN-gamma), ${ }^{4}$ so as to complement strategies such as staining and culture for acid-fast bacilli. ${ }^{2}$ In a systematic review of 63 studies ADA was characterised by positive likelihood ratio 9.03 (95\% confidence interval (CI) 7.19 to 11.35 ), negative likelihood ration 0.10 (95\% CI 0.07 to 0.14 ) for diagnosis of tuberculous pleural effusion. ${ }^{3}$ Correspondingly, in a systematic review of 22 studies, diagnostic accuracy of IFN-gamma was characterised by positive likelihood ration amounting to 23.45 (95\% CI 17.31 to 31.78 ), and negative likelihood ratio 0.11 (95\% CI 0.07 to 0.16$).{ }^{4}$ In the setting of tuberculous effusion prevalence of $5 \%$, post-test probability of a negative ADA test has been estimate to be $0.4 \%$, increasing to $2.4 \%$ where tuberculous pleural effusion has a $25 \%$ prevalence. For INF-gamma, corresponding post-test probabilities are $0.22 \%$ and $1.2 \%$ respectively.

OSCAR MP JOLOBE

Manchester Medical Society,

Manchester 